\title{
A dynamic model for hysteresis in magnetostrictive devices
}

M. Trapanese, V. Franzitta, and A. Viola

Citation: Journal of Applied Physics 115, 17D141 (2014); doi: 10.1063/1.4868708

View online: https://doi.org/10.1063/1.4868708

View Table of Contents: http://aip.scitation.org/toc/jap/115/17

Published by the American Institute of Physics

\section{Articles you may be interested in}

A general one-dimension nonlinear magneto-elastic coupled constitutive model for magnetostrictive materials AIP Advances 5, 107201 (2015); 10.1063/1.4933024

Enhanced sensitivity in magnetoelectric current-sensing devices with frequency up-conversion mechanism by modulating the magnetostrictive strain

Journal of Applied Physics 115, 17 E505 (2014); 10.1063/1.4862081

Magnetostrictive vibrations model of a three-phase transformer core and the contribution of the fifth harmonic in the grid voltage

Journal of Applied Physics 115, 17 A316 (2014); 10.1063/1.4863931

Improvement of force factor of magnetostrictive vibration power generator for high efficiency

Journal of Applied Physics 117, 17B508 (2015); 10.1063/1.4907237

Free energy model for hysteresis in magnetostrictive transducers

Journal of Applied Physics 93, 458 (2003); 10.1063/1.1524312

Physical interpretation of eddy current losses in ferromagnetic materials. I. Theoretical considerations Journal of Applied Physics 57, 2110 (1985); 10.1063/1.334404

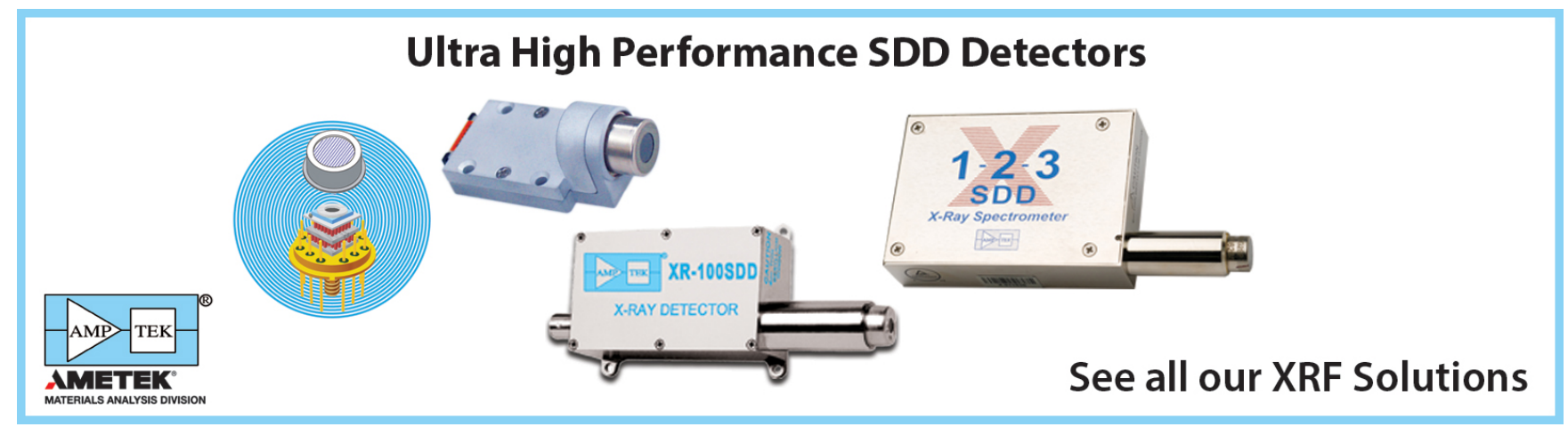




\title{
A dynamic model for hysteresis in magnetostrictive devices
}

\author{
M. Trapanese, ${ }^{\text {a) }}$ V. Franzitta, and A. Viola \\ DEIM, Palermo University, Palermo, I-90128 Palermo, Italy
}

(Presented 5 November 2013; received 23 September 2013; accepted 26 December 2013; published online 26 March 2014)

\begin{abstract}
In this paper, a dynamic model for the description and design of hysteresis in magnetostrictive devices is presented. The model is based on Preisach theory and its dynamic extension. A procedure for determining the Preisach distribution function is given. This procedure is based on neural networks. The model is able to reconstruct both the magnetization relation and the fieldstrain relation. The model is validated through comparison and prediction of data collected from a typical Terfenol-D sample and a novel experimental technique dedicated to the validation of dynamic models is proposed. (C) 2014 AIP Publishing LLC. [http://dx.doi.org/10.1063/1.4868708]
\end{abstract}

\section{INTRODUCTION AND MOTIVATION}

Recently, magnetostrictive materials have been proposed as active materials to be used in several energy harvesting technology. ${ }^{1}$ In this kind of application, the mechanical stress applied by an external source (that could be cars, walking or running persons, vibrations induced on a structure, etc.) is the primary energy source that could be transformed in electrical energy. Because of the fact that an energy source of this type is inherently stochastic, the working condition of the material is highly dynamic and nonlinear. As a result, in this kind of condition, static models of magnetostrictive materials are usually not very accurate and may be unreliable to develop a sufficiently accurate design of the energy harvesting devices. Moreover, the presence of hysteresis in both the mechanical and magnetic characteristics requires accurate mathematical modeling in order to correctly foresee the behaviour of real materials used in control systems or in electrical machines, to simplify the design of such controllers as well as to predict with acceptable accuracy electromagnetic fields in such devices. ${ }^{2}$

Therefore, the motivation of the work here reported is to propose a mathematical model able to predict the dynamic behaviour of magnetostrictive materials used in energy applications. Several authors have already proposed mathematical models of magnetostrictive materials; ${ }^{3-6}$ however, most of the proposed approaches are based on static models. This paper presents a mathematical model of hysteresis in magnetostrictive materials based on the use of Dynamic Preisach hysteresis model (DPM) which is able to model the dynamic conditions of the system. A model identification procedure based on a previous developed approach ${ }^{7-9}$ is given. The model is tested by using some published data on Terfenol D. Furthermore, a novel experimental technique based on acoustic emission is proposed to test model.

The paper presents in Sec. II the dynamic Preisach Model used to model the magnetostrictive behaviour, in Sec. III the identification procedure is proposed, in Sec. IV the

\footnotetext{
a) Author to whom correspondence should be addressed. Electronic mail: marco.trapanese@unipa.it. Fax: +39-091488452.
}

experimental setup and the experimental results are discussed, and in Sec. V some results are drawn.

\section{HYSTERESIS IN MAGNETOSTRICTIVE MATERIALS}

It is known that in most ferromagnetic materials magnetic properties and mechanical properties are tightly interrelated. Generally speaking, an alteration of the magnetization level of a ferromagnetic sample is usually linked to a variation in both shape and dimension and vice versa. Materials, where this effect is large, are called magnetostrictive materials. This effect is usually exploited in sensors. More recently, the fact that mechanical stress induces a change in the level of magnetization of a sample and therefore this change in magnetization induces an electromotive force (emf) has been proposed as a tool to convert environmental vibration into electric energy. This kind of application faces a highly stochastic and nonlinear environment and is based on the coupling of the mechanical and magnetic behaviour.

As a result, in order to model such a behaviour, a simplified version of DPM has been used. DPM is a modification of Classical Preisach Model (CPM) introduced to describe dynamic features of magnetic systems.

Our model can be described as follows:

(1) The magnetization of the material is obtained by using DPM;

(2) The magnetization of the material is the input of the Preisach model which describes the strain $\lambda$ of the material.

A complete description of the DPM can be found in Ref. 10. Below, we will give only the mathematical detail needed for the comprehension of this paper. In DPM, the magnetization $\mathrm{M}(\mathrm{t})$ depends on time $\mathrm{t}$ and varies accordingly to the following equation:

$$
\mathrm{M}(\mathrm{t})=\mathrm{M}_{\mathrm{s}} \int_{0}^{\infty} \mathrm{dh} \mathrm{h}_{\mathrm{c}} \int_{-\infty}^{b\left(h_{c}\right)} \mathrm{p}\left(\mathrm{h}_{\mathrm{c}}, \mathrm{h}_{\mathrm{m}}\right) \cdot \varphi\left(\mathrm{h}_{\mathrm{c}}, \mathrm{h}_{\mathrm{m}}, \mathrm{t}\right) \mathrm{dh}_{\mathrm{m}},
$$

where $M(t)$ is the magnetization, $M_{s}$ is the saturation magnetization (i.e., the maximum value achievable by 
magnetization), $\mathrm{p}\left(\mathrm{h}_{\mathrm{c}}, \mathrm{h}_{\mathrm{m}}\right)$ is the Preisach Density Function (PDF), and $h_{c}$ and $h_{m}$ are the parameters that describe the values of the switching fields of each hysteron. $\varphi\left(\mathrm{h}_{\mathrm{c}}, \mathrm{h}_{\mathrm{m}}, \mathrm{t}\right)$ describes the state of each elementary Preisach Model loop at the time $\mathrm{t}$ and introduces a dependence of the maximum value of each hysteron on time, $\varphi\left(\mathrm{h}_{\mathrm{c}}, \mathrm{h}_{\mathrm{m}}, \mathrm{t}\right)$ varies according to the following equation:

$$
\frac{\partial \varphi\left(h_{m}, h_{c}, t\right)}{\partial t}= \begin{cases}k \cdot\left[H(t)-\left(h_{m}+h_{c}\right)\right], & \text { if } H(t)\rangle\left(h_{m}+h_{c}\right) \\ k \cdot\left[H(t)-\left(h_{m}-h_{c}\right)\right], & \text { if } H(t)\left\langle\left(h_{m}-h_{c}\right)\right. \\ 0 & \text { elsewhere, }\end{cases}
$$

where $\mathrm{k}$ is an unknown to be determined parameter that must be chosen so to optimize the description of the material whose hysteresis description is sought. DPM becomes equivalent to CPM if the parameter $\mathrm{k}$ becomes infinite, because, in this case, the function $\varphi\left(\mathrm{h}_{\mathrm{c}}, \mathrm{h}_{\mathrm{m}}, \mathrm{t}\right)$ can assume only the values -1 and +1 . The parameter $\mathrm{k}$ quantifies the finite rate of the switching of the hysterons of DPM.

From what said above, it can be deducted that the total number of parameters needed in order to identify a generic material in DPM is equal to the sum of the number of parameters of PDF and $\mathrm{k}$.

The magnetization computed in Eq. (1) is the input for the calculation of the strain of the material, which can be computed as follows:

$$
\begin{aligned}
\lambda(\mathrm{M}(\mathrm{t}))= & \lambda_{\text {rev }}(\mathrm{M}(\mathrm{t})) \\
& +\frac{1}{2} \int_{-\mathrm{M}_{\mathrm{s}}}^{+\mathrm{M}_{\mathrm{s}}} \mathrm{dh}_{\mathrm{c}} \int_{-\mathrm{M}_{\mathrm{s}}}^{h c} \mathrm{q}\left(\mathrm{h}_{\mathrm{c}}, \mathrm{h}_{\mathrm{m}}\right) \cdot \varphi\left(\mathrm{h}_{\mathrm{c}}, \mathrm{h}_{\mathrm{m}}\right) \mathrm{dh}_{\mathrm{m}},
\end{aligned}
$$

where $\lambda$ is the strain, $\lambda_{\text {rev }}$ is the reversible part of magnetostriction, and q is the PDF for the model of strain.

The complete dynamic model of a magnetostrictive material is given by Eqs. (1)-(3). In order to identify, the model one must identify the Preisach density functions $\mathrm{p}$ and $\mathrm{q}$ used in Eqs. (1) and (3), this means that the parameters of the functions $\mathrm{p}$ and $\mathrm{q}$ must be explicitly given. The identification of PDFs can be performed in several ways ${ }^{2}$ and generally speaking a numerical description of PDFs is obtained. Such an expression of PDFs can be computationally heavy and difficult to be used for describing and designing a harvesting device. As a result, it is preferable to use analytical expression of PDFs and adapt them to experimental curve. In this paper, two Gaussian PDFs have been used. This approach leads to the identification procedure below sketched.

\section{IDENTIFICATION PROCEDURE}

The fundamental idea of the identification procedure is to train a neural network with some $a$ priori built hysteresis curves (whose parameters are known) and to use this trained network to find the best parameters that describe some unknown hysteresis curve (Fig. 1). The identification procedure consists of four steps: (A) network training; (B)

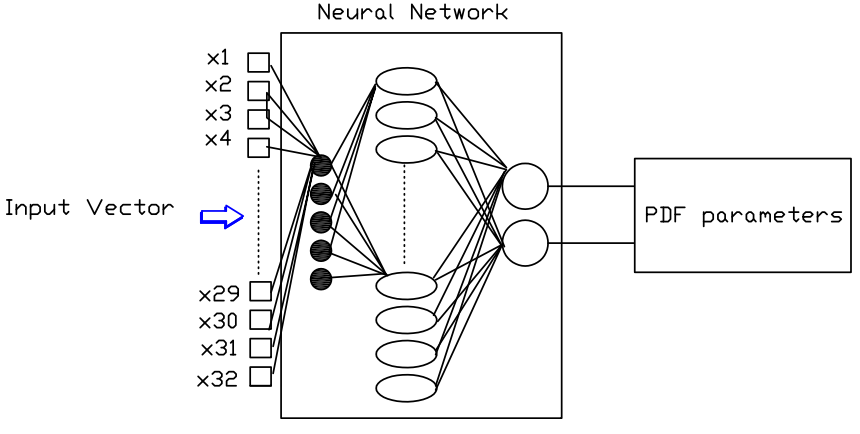

FIG. 1. Conceptual scheme of the identification procedure.

submission of test set; (C) output of the results; (D) experimental verification.

\section{A. Network training}

The neural network that has been chosen is a three layer neural network: the first layer consists of 5 linear neurons, the second layer of 25 neurons with hyperbolic tangent sigmoid function, and the output layer consists of 5 linear neurons. The input is made up of 100 units which describe the magnetic and strain hysteresis curve numerically computed by using some known parameters and five other units which are the parameters used to compute the hysteresis curves. The first layer makes a linear reduction from the high dimension input space to the lower 5-dimension space: the output of the first layer is therefore the feature vector that sums up the most relevant characteristics of the input space and is strictly connected to five relevant properties of the hysteresis loop: remanence, coercive field, and slopes in correspondence of some points of the loop. The 25 neurons of the second layer allow to store a number of parametric curves at least equal to 500. The output layer is made up of five neurons which are the free parameters of the PDFs of the model that most suitably permit to produce the inputs.

The training set consists of one hundred vectors. Each input vector consists of 100 coordinates and 5 target vectors. The first one hundred coordinates are the ordinates of magnetic and strain hysteresis curve. The excitation amplitude is the same in all cases. The last five coordinates are the target vectors.

\section{B. Submission of the test set}

The first test is made up of the ordinates of two numerical hysteresis loops, which were obtained by making the parameters of the Preisach function different from those of the training set.

A second test set was created by using a real hysteresis loop.

In both cases, the abscissa field is equal to the one used in the training phase.

\section{Output of the results}

The output of the network consists, as said above, of five parameters. They represent the parameters of the two Gaussian distribution functions which are associated to the presented loops and the dynamic parameter $\mathrm{k}$. 


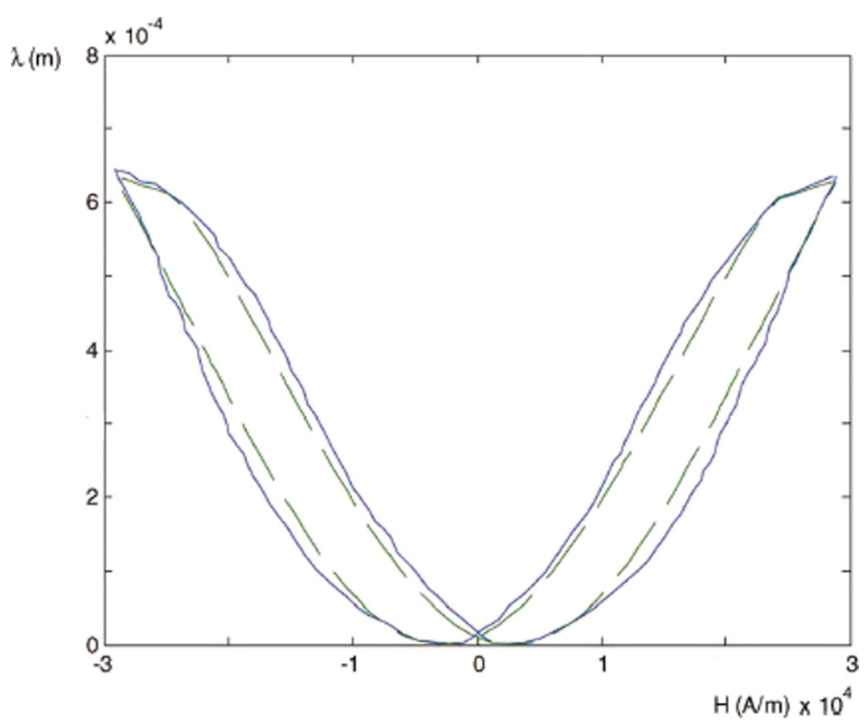

FIG. 2. Magnetostriction magnetization characteristics. Continuous line represents the reconstructed curve and the dotted line is the experimental line.

\section{NUMERICAL AND EXPERIMENTAL VERIFICATION}

In order to verify the ability of the trained neural network to obtain the exact parameters of the submitted curves two tests were performed: in the first one the magnetic hysteresis curve of TERFENOL was submitted to the network and in the second one the magneto-mechanical curve of TERFENOL was derived in terms of the parameters given by the network,

In order to evaluate the quality of the obtained reconstruction, the index defined in Ref. 7 was used.

Terfenol magnetic and strain hysteresis reconstruction

The first test consisted in submitting TERFENOL magnetic hysteresis and verifying the ability of the network to find parameters useful for reconstructing terfenol hysteresis curve.

The curve was obtained by using a Gaussian PDF for the magnetization in static condition. The error between the experimental curve and the reconstructed curve is in the range of $10 \%$.

Finally, in Fig. 2, the strain hysteresis curve is shown. It can be seen how the model is able to reconstruct the strain curve within a precision of $15 \%$.

In order to verify the capability of the developed approach to give information on the dynamic features of the material, we have developed the following procedure:

(1) the strain response to an external sinusoidal magnetic field was computed by using Eqs. (1)-(3);

(2) the harmonic content of the response was computed by performing a fast Fourier transform on the obtained strain;

(3) the harmonic contents was detected in terms of noise emissions.

A simple experimental test was performed, which consisted in applying a sinusoidal field to a rod shaped sample and detecting the acoustic emissions of this sample. In Fig. 3, it is shown how the model was able to predict the first three odd harmonics (the fundamental harmonic and the third and fifth harmonic) of the acoustic emission of the sample. Unfortunately, the model predicted also the presence of two

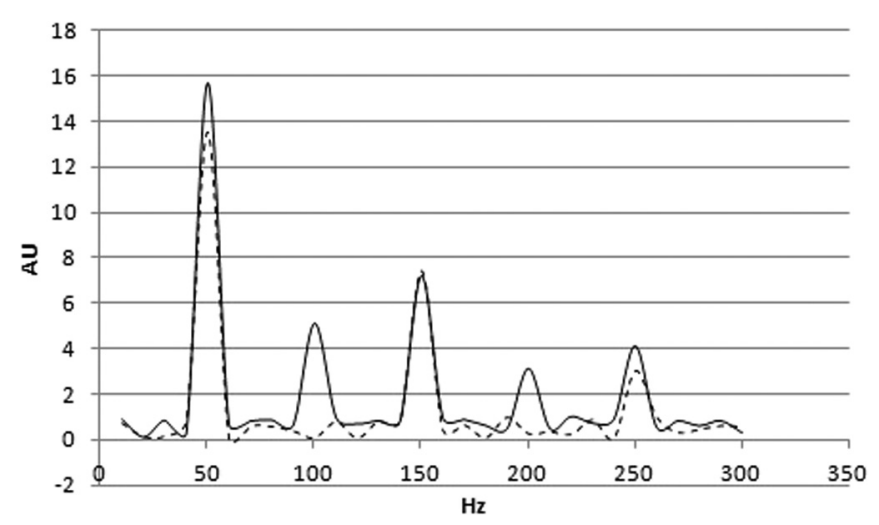

FIG. 3. A comparison between the reconstructed spectrum (continuous line) and the experimental spectrum (dotted line) of the acoustic emission of a rod of Terfenol D.

even harmonics that were not detected. This fact was caused by the fact the mathematical model shows some minor loops whose symmetry is described in terms of even harmonics. This type of minor loops was not experimentally detected.

\section{CONCLUSION}

This paper presents a method based on neural networks for the identification of the parameters of DPM able to reconstruct the behaviour of terfenol. The fundamental idea of this method is to identify the parameters of a material by using a neural network trained by a collection of hysteresis curves, whose Preisach model is known. The numerical results show that the method can reconstruct and find the parameters of hysteresis curves that were not used in the training phase. Finally, an experimental procedure based on the detection of acoustic emissions to check the validity of the dynamic model was introduced and partially validated.

\section{ACKNOWLEDGMENTS}

This work was funded by IMPETUS project.

${ }^{1}$ H. Zang, Appl. Phys. Lett. 98, 232505 (2011).

${ }^{2} \mathrm{C}$. Natale, F. Velardi, and C. Visone, "Identification and compensation of Preisach hysteresis models for magnetostrictive actuators," Physica B 306, 161-165 (2001)

${ }^{3}$ L. Dupré, M. De Wulf, D. Makaveev, V. Permiakov, and J. Melkebeek, "Preisach modelling of magnetization and magnetostriction processes in laminated SiFe alloys," J. Appl. Phys. 93(10), 6629 (2003).

${ }^{4}$ D. C. Jiles, "Hysteresis models: Nonlinear magnetism on length scales from the atomistic to the macroscopic," J. Magn. Magn. Mater. 242-245, 116-124 (2002).

${ }^{5}$ F. T. Calkins, R. C. Smith, and A. B. Flatau, "Energy-based hysteresis model for magnetostrictive transducers," IEEE Trans. Magn. 36, 429-439 (2000).

${ }^{6} \mathrm{~K}$. Linnemann, S. Klinkel, and W. Wagner, "A constitutive model for magnetostrictive and piezoelectric materials," Int. J. Solids Struct. 46, 1149-1166 (2009).

${ }^{7}$ M. Cirrincione, R. Miceli, G. R. Galluzzo, and M. Trapanese, "A novel neural approach to the determination of the distribution function in magnetic Preisach systems," IEEE Trans. Magn. 40(4), 2131-2133 (2004).

${ }^{8} \mathrm{M}$. Trapanese, "Identification of parameters of the Jiles-Atherton model by neural networks," J. Appl. Phys. 109(7), 07D355 (2011).

${ }^{9} \mathrm{M}$. Trapanese, "Identification of the parameters of reduced vector Preisach model by neural networks," IEEE Trans. Magn. 44(11), 3197-3200 (2008).

${ }^{10}$ G. Bertotti, IEEE Trans. Magn. 28, 2599 (1992). 